Pacific Journal of Mathematics

COHOMOLOGICAL DIMENSION OF DISCRETE MODULES 


\title{
COHOMOLOGICAL DIMENSION OF DISCRETE MODULES OVER PROFINITE GROUPS
}

\author{
JUAN José MaRTÍNeZ
}

\begin{abstract}
The main purpose of this note is to show that the finiteness of the cohomological dimension of a discrete module is closely related to the finiteness of its injective dimension. Moreover, a sufficient condition for the finiteness of the cohomological dimension is given. Both results are proved making a heavy use of the theory of cohomological triviality for finite groups.
\end{abstract}

The reader is referred to [3] for a treatment of profinite cohomo$\log$.

Throughout this note, $G$ is a profinite group. As usual, the cohomology of $G$ is denoted by $H(G$, ).

Recall that, if $A$ is a discrete $G$-module, the infimum of the (set of) nonnegative integers $r$ such that $H^{n}(S, A)=0$, for any integer $n>r$ and any closed subgroup $S$ of $G$, is called the cohomological dimension of $A$, and is denoted by $c d(G, A)$. If $S$ is a closed subgroup of $G, H^{n}(S, A) \cong \lim H^{n}(V, A)$, where $V$ runs through all open subgroups of $G$ containing $S$ [3, Chap. I, Proposition 8, p. I-9]. Hence, if $H^{n}(V, A)=0$ for every open subgroup $V$ of $G$, then $H^{n}(S, A)=0$ for every closed subgroup $S$ of $G$.

In this paper, a discrete module is called injective only when it is injective in the corresponding category of discrete modules. If $A$ is injective, it is well-known that $c d(G, A)=0$, because, for instance, $A$ is $V$-injective for all open subgroups $V$ of $G$. Finally, recall that the injective dimension of $A$, denoted by $i d(G, A)$, is the least length of an injective resolution of $A$.

The connection between cohomologically trivial modules over finite groups [2, Chap. IX, $\S 3$, p. 148] and discrete modules of cohomological dimension zero over profinite groups was observed, and used, by Tate in his duality theory for profinite cohomology [3, Annexe au Chapitre I, p. I-79]. Tate's observation is quoted, for future reference, in the following.

Lemma 1. Let $A$ be a discrete G-module. Then, $c d(G, A)=0$ if, and only if, for every open, normal subgroup $U$ of $G$, the $G / U$-module $A^{U}$ is cohomologically trivial.

Proof. See [3, Annexe au Chapitre I, Lemme 1, p. I-82]. Notice that $G / U$ is a finite group, because $G$ is compact and $U$ is open.

The Nakayama-Tate criterion for cohomological triviality takes 
the following form, in the cohomology theory of profinite groups.

Proposition 2. Let $A$ be a discrete G-module. If there exists a positive integer $q$ such that $H^{q}(V, A)=H^{q+1}(V, A)=0$ for all open subgroups $V$ of $G$, then $\operatorname{cd}(G, A)<q$.

Proof. Since $A$ embeds in an injective, whose cohomological dimension is zero, by repeated applications of dimension-shifting it suffices to consider the case $q=1$. Let $U$ be an open, normal subgroup of $G$. If $V$ is any subgroup of $G$ containing $U$, the HochschildSerre spectral sequence of the $V / U$-module $A^{U}$ yields the exact sequence for low degrees

$$
\begin{aligned}
0 \longrightarrow H^{1}\left(V / U, A^{U}\right) \longrightarrow H^{1}(V, A) \longrightarrow H^{1}(U, A)^{V / U} & \longrightarrow H^{2}\left(V / U, A^{U}\right) \longrightarrow H^{2}(V, A) .
\end{aligned}
$$

Since $U$ is open, so is $V$, and thus, $H^{1}(U, A)=H^{1}(V, A)=H^{2}(V, A)=$ 0 . Therefore, $H^{1}\left(V / U, A^{U}\right)=H^{2}\left(V / U, A^{U}\right)=0$, and applying the Nakayama-Tate criterion [2, Chap. IX, Théorème 8, p. 152], the $G / U$ module $A^{U}$ is cohomologically trivial. By (1), the proof is complete.

The main result of this paper can be stated as follows.

THEOREM 3. Let $A$ be a discrete G-module, and let $q$ be a positive integer. Then, $i d(G, A) \leqq q$ if, and only if, $c d(G, A) \leqq q$ and $H^{q}(U, A)$ is a divisible abelian group for every open, normal subgroup $U$ of $G$.

Proof. Assume the assertion true for $q-1$, with $q>1$. If $i d(G, A) \leqq q, A$ has an injective resolution of length $\leqq q$, say

$$
0 \longrightarrow A \stackrel{e}{\longrightarrow} X_{0} \stackrel{d_{0}}{\longrightarrow} X_{1} \longrightarrow \cdots \longrightarrow X_{q-1} \stackrel{d_{q-1}}{\longrightarrow} X_{q} \longrightarrow 0 \text {. }
$$

If $B=$ Coker $e$ and $f: X_{0} \rightarrow B$ is the canonical morphism, the sequence of discrete $G$-modules

$$
0 \longrightarrow A \stackrel{e}{\longrightarrow} X_{0} \stackrel{f}{\longrightarrow} B \longrightarrow 0
$$

is exact. Since $\operatorname{cd}\left(G, X_{0}\right)=0$ (injectivity of $X_{0}$ ), from the corresponding cohomology sequence it follows that

$$
H^{n}(S, B) \cong H^{n+1}(S, A)
$$

for any positive integer $n$ and any closed subgroup $S$ of $G$. Therefore, it is enough to prove that $c d(G, B) \leqq q-1$, and that $H^{q-1}(U, B)$ is divisible for all open, normal subgroups $U$ of $G$. By the induction hypothesis, this follows from showing that $i d(G, B) \leqq q-1$. In fact, if $e^{\prime}: B \rightarrow X_{1}$ is the morphism induced by $d_{0}: X_{0} \rightarrow X_{1}$, then $\operatorname{Ker} e^{\prime}=0$ and $\operatorname{Im} e^{\prime}=\operatorname{Im} d_{0}$. Thus, the sequence 


$$
0 \longrightarrow B \stackrel{e^{\prime}}{\longrightarrow} X_{1} \stackrel{d_{1}}{\longrightarrow} X_{2} \longrightarrow \cdots \longrightarrow X_{q-1} \stackrel{d_{q-1}}{\longrightarrow} X_{q} \longrightarrow 0
$$

is exact.

Reciprocally, if $c d(G, A) \leqq q$, let

$$
0 \longrightarrow A \stackrel{g}{\longrightarrow} Q \stackrel{h}{\longrightarrow} C \longrightarrow 0
$$

be an exact sequence of discrete $G$-modules, with $Q$ injective. Then, $c d(G, C) \leqq q-1$, because

$$
H^{n}(S, C) \cong H^{n+1}(S, A)
$$

for all positive integers $n$ and all closed subgroups $S$ of $G$. By the same reason, if $H^{q}(U, A)$ is divisible for every open, normal subgroup $U$ of $G$, then so is $H^{q-1}(U, C)$. Hence, by induction, $C$ admits an injective resolution of length $\leqq q-1$, say

$$
0 \longrightarrow C \stackrel{i}{\longrightarrow} Y_{0} \stackrel{d_{0}}{\longrightarrow} Y_{1} \longrightarrow \cdots \longrightarrow Y_{q-2} \stackrel{d_{q-2}}{\longrightarrow} Y_{q-1} \longrightarrow 0 \text {. }
$$

Since $\operatorname{Ker} i h=\operatorname{Ker} h$ and $\operatorname{Im} i h=\operatorname{Im} i$, the sequence

$$
0 \longrightarrow A \stackrel{g}{\longrightarrow} Q \stackrel{i h}{\longrightarrow} Y_{0} \stackrel{d_{0}}{\longrightarrow} Y_{1} \longrightarrow \cdots \longrightarrow Y_{q-2} \stackrel{d_{q-2}}{\longrightarrow} Y_{q-1} \longrightarrow 0
$$

is exact, and so $i d(G, A) \leqq q$.

It remains to prove the assertion for $q=1$.

Let

$$
0 \longrightarrow A \longrightarrow X_{0} \longrightarrow X_{1} \longrightarrow 0
$$

be an exact sequence of discrete $G$-modules, where $X_{0}$ and $X_{1}$ are injectives. Since $c d\left(G, X_{0}\right)=c d\left(G, X_{1}\right)=0$, passing to cohomology it follows that $c d(G, A) \leqq 1$, and that the connecting operator $\partial_{S}: X_{1}^{S} \rightarrow$ $H^{1}(S, A)$ is an epimorphism for all closed subgroups $S$ of $G$. But, if $D$ is any injective, discrete $G$-module and $U$ is any open, normal subgroup of $G$, it is easy to check that $D^{U}$ is an injective $G / U$-module, whence [2, Chap. IX, Lemme 7, p. 153] implies $D^{U}$ is divisible. Therefore, as the image of a divisible group, $H^{1}(U, A)$ is divisible for all open, normal subgroups $U$ of $G$.

Reciprocally, suppose $c d(G, A) \leqq 1$, and let

$$
0 \longrightarrow A \longrightarrow Y_{0} \longrightarrow Y_{1} \longrightarrow 0
$$

be an exact sequence of discrete $G$-modules, with $Y_{0}$ injective. Since $c d\left(G, Y_{0}\right)=0$, taking cohomology it follows that $c d\left(G, Y_{1}\right)=0$, and that the sequence of abelian groups

$$
Y_{0}^{S} \longrightarrow Y_{1}^{S} \stackrel{\partial_{S}}{\longrightarrow} H^{1}(S, A) \longrightarrow 0
$$


is exact for all closed subgroups $S$ of $G$. If $U$ is an open, normal subgroup of $G$, $\operatorname{Ker} \partial_{U}$ is divisible, because so is $Y_{0}^{U}$. Therefore, if $\operatorname{Im} \partial_{U}=H^{1}(U, A)$ is divisible, then Dom $\partial_{U}=Y_{1}^{U}$ is also divisible, and the proof is complete applying to $Y_{1}$ the following.

Proposition 4. Let $A$ be a discrete G-module. If $c d(G, A)=0$, and $A^{U}$ is a divisible abelian group for every open, normal subgroup $U$ of $G$, then $A$ is injective.

Proof. Recall that the category of discrete $G$-modules has injective envelopes for each of its objects. Since $(Z[G / U])_{U}$, where $U$ runs through all open, normal subgroups of $G$, is a family of generators, this result can be obtained by using a general theorem from category theory, due to Mitchell [1, Chap. III, Theorem 3.2, p. 89].

Let $f: A \rightarrow Q$ be an injective envelope of $A$ (in the category of discrete $G$-modules). If $C=\operatorname{Coker} f$ and $g: Q \rightarrow C$ is the canonical morphism, the sequence of discrete $G$-modules

$$
0 \longrightarrow A \stackrel{f}{\longrightarrow} Q \stackrel{g}{\longrightarrow} C \longrightarrow 0
$$

is exact. Thus, if $U$ is an open, normal subgroup of $G$, the sequence of $G / U$-modules

$$
0 \longrightarrow A^{U} \stackrel{f^{U}}{\longrightarrow} Q^{U} \stackrel{g^{U}}{\longrightarrow} C^{U} \longrightarrow 0
$$

is exact, because $c d(G, A)=0$. Since $Q^{U}$ is an injective $G / U$-module and $R \cap \operatorname{Im} f^{U}=R \cap \operatorname{Im} f$ for any sub-G/U-module $R$ of $Q^{U}$ (because, regarding $R$ as a $G$-module, $U$ operates trivially on $R$ ), $f^{U}: A^{U} \rightarrow Q^{U}$ is an injective envelope of $A^{U}$ (in the category of $G / U$-modules). On the other hand, since $c d(G, A)=0, A^{U}$ is a cohomologically trivial $G / U$-module, by (1). Thus, $A^{U}$ is $G / U$-injective [2, Chap. IX, Théorème 10, p. 154], and hence, $C^{U}=0$ [1, Chap. III, Proposition 2.5, p. 88]. Since $C=\cup C^{U}, C=0$, whence the result.

Corollary 5. Let $A$ be a discrete G-module, and let $r$ be a nonnegative integer. If $c d(G, A) \leqq r$, then $i d(G, A) \leqq r+1$.

Proof. Take $q=r+1$ in (3).

This result can be applied to profinite groups of finite dimension, as follows.

COROLlaRY 6. Let $r$ be a nonnegative integer. The following statements are true:

( i) If $p$ is a prime number and $c d_{p}(G) \leqq r$, then $i d(G, A) \leqq$ $r+1$ for all discrete $G$-modules $A$ which are $p$-primary abelian groups. 
(ii) If $c d(G) \leqq r$, then $i d(G, A) \leqq r+1$ for all discrete $G$-modules $A$ which are torsion abelian groups.

(iii) If $\operatorname{scd}(G) \leqq r$, then $i d(G, A) \leqq r+1$ for all discrete $G$-modules $A$. ules $A$.

(iv) If $c d(G) \leqq r$, then $i d(G, A) \leqq r+2$ for all discrete $G$-mod-

Proof. Applying [3, Chap. I, Proposition 14, p. I-20] and [3, Chap. I, Proposition 11, p. I-17], the following three equivalences are clear:

(i) $c d_{p}(G) \leqq r$ if, and only if, $c d(G, A) \leqq r$ for all $p$-primary, discrete $G$-modules $A$.

(ii) $\quad c d(G) \leqq r$ if, and only if, $c d(G, A) \leqq r$ for all torsion, discrete $G$-modules $A$.

(iii) $\operatorname{scd}(G) \leqq r$ if, and only if, $c d(G, A) \leqq r$ for all discrete $G$ modules $A$.

Finally, (6, iv) is clear by [3, Chap. I, Proposition 13, p. 1-19].

\section{REFERENCES}

1. B. Mitchell, Theory of Categories, Pure and applied mathematics 17, Academic Press, New York, 1965.

2. J-P. Serre, Corps Locaux, Actualités scientifiques et industrielles 1296, Hermann, Paris, 1962.

3. Cohomologie Galoisiene, Lecture notes in mathematics 5, Springer-Verlag, Berlin, 1965.

Received August 14, 1972 and in revised form December 18, 1972.

Universidad de Buenos Aires 



\section{PACIFIC JOURNAL OF MATHEMATICS}

\section{EDITORS}

RICHARD ARENS (Managing Editor) University of California

Los Angeles, California 90024

R. A. Beaumon'T

University of Washington Seattle, Washington 98105
J. Dugundj1*

Department of Mathematics University of Southern California Los Angeles, California 90007

D. Gilbarg and J. Milgram Stanford University Stanford, California 94305

\section{ASSOCIATE EDITORS}

E. F. BECKENBACH

B. H. NeUMANN

F. WOLF

K. YOSHIDA

\section{SUPPORTING INSTITUTIONS}

UNIVERSITY OF BRITISH COLUMBIA CALIFORNIA INSTITUTE OF TECHNOLOGY UNIVERSITY OF CALIFORNIA MONTANA STATE UNIVERSITY UNIVERSITY OF NEVADA NEW MEXICO STATE UNIVERSITY OREGON STATE UNIVERSITY UNIVERSITY OF OREGON OSAKA UNIVERSITY

\section{UNIVERSITY OF SOUTHERN CALIFORNIA STANFORD UNIVERSITY UNIVERSITY OF TOKYO UNIVERSITY OF UTAH WASHINGTON STATE UNIVERSITY UNIVERSITY OF WASHINGTON AMERICAN MATHEMATICAL SOCIETY NAVAL WEAPONS CENTER}

* C. R. DePrima California Institute of Technology, Pasadena, CA 91109, will replace J. Dugundji until August 1974. 


\section{Pacific Journal of Mathematics}

\section{Vol. 49, No. $1 \quad$ May, 1973}

A. Bigard, Free lattice-ordered modules ...........................

Richard Bolstein and Warren R. Wogen, Subnormal operators in strictly cyclic

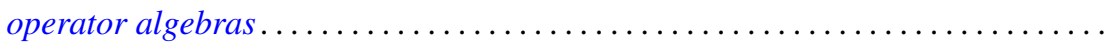

Herbert Busemann and Donald E. Glassco, II, Irreducible sums of simple

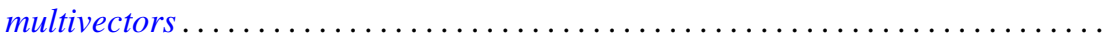

W. Wistar (William) Comfort and Victor Harold Saks, Countably compact groups

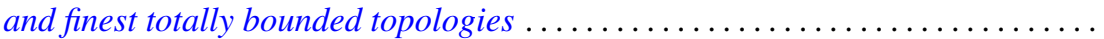

Mary Rodriguez Embry, Maximal invariant subspaces of strictly cyclic operator

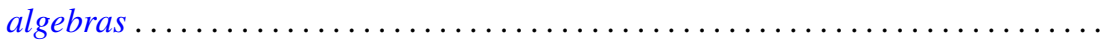

Ralph S. Freese and James Bryant Nation, Congruence lattices of semilattices......

Ervin Fried and George Grätzer, A nonassociative extension of the class of

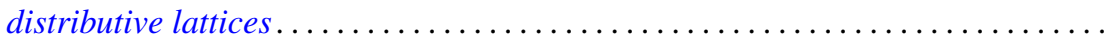

John R. Giles and Donald Otto Koehler, On numerical ranges of elements of locally

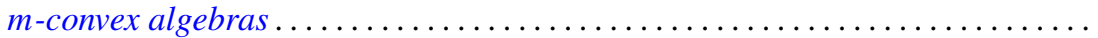

David A. Hill, On dominant and codominant dimension of $\mathrm{QF}-3$ rings ........ John Sollion Hsia and Robert Paul Johnson, Round and Pfister forms over $R(t) \ldots$ I. Martin (Irving) Isaacs, Equally partitioned groups . . . . . . . . . . . . . .

Athanassios G. Kartsatos and Edward Barry Saff, Hyperpolynomial approximation of solutions of nonlinear integro-differential equations.

Shin'ichi Kinoshita, On elementary ideals of $\theta$-curves in the 3-sphere and 2-links in the 4-sphere

Ronald Brian Kirk, Convergence of Baire measures

R. J. Knill, The Seifert and Van Kampen theorem via regular covering spaces ..

Amos A. Kovacs, Homomorphisms of matrix rings into matrix rings ..

Young K. Kwon, HD-minimal but no $H D$-minimal ..........

Makoto Maejima, On the renewal function when some of the mean renewal lifetimes are infinite

Juan José Martínez, Cohomological dimension of discrete modules over profinite groups.

W. K. Nicholson, Semiperfect rings with abelian group of units

Louis Jackson Ratliff, Jr., Three theorems on imbedded prime divisors of principal ideals.

Billy E. Rhoades and Albert Wilansky, Some commutants in $B(c)$ which are almost matrices

John Philip Riley Jr., Cross-sections of decompositions . . .

Keith Duncan Stroyan, A characterization of the Mackey uniformity $m\left(L^{\infty}, L^{1}\right)$ for

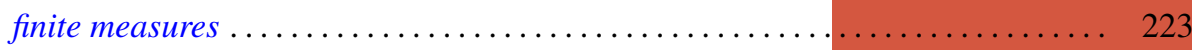

Edward G. Thurber, The Scholz-Brauer problem on addition chains . . . . . . . . . 229

Joze Vrabec, Submanifolds of acyclic 3-manifolds ............

Philip William Walker, Adjoint boundary value problems for compactified singular

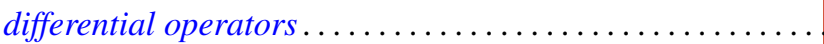

